OPEN ACCESS

Edited by:

Jianwei Su,

Anhui University, China

Reviewed by:

Kun Zhang,

Tongji University, China

Dalong Ni,

Shanghai Jiao Tong University, China

${ }^{*}$ Correspondence:

Feixiang Chen

ChenfeixiangEcnu@163.com

Specialty section: This article was submitted to Nanoscience,

a section of the journal

Frontiers in Chemistry

Received: 14 January 2022

Accepted: 01 February 2022

Published: 21 February 2022

Citation:

Chen $F$ and Song $T$ (2022) AuPt Bimetallic Nanozymes for Enhanced

Glucose Catalytic Oxidase.

Front. Chem. 10:854516

doi: 10.3389/fchem.2022.854516

\section{AuPt Bimetallic Nanozymes for Enhanced Glucose Catalytic Oxidase}

\author{
Feixiang Chen ${ }^{1 *}$ and Tianlin Song ${ }^{2}$ \\ ${ }^{1}$ Shanghai Key Laboratory of Green Chemistry and Chemical Processes, School of Chemistry and Molecular Engineering, East \\ China Normal University, Shanghai, China, ${ }^{2}$ Tongji University Cancer Center, Shanghai Tenth People's Hospital, Tongji University \\ School of Medicine, Shanghai, China
}

Au metal nanoparticles as artificial nanozymes have attracted wide interest in biotechnology due to high stability and easy synthesis. Unfortunately, its catalytic activity is limited by the uniform surface electron distribution, fundamentally affecting the oxidation efficiency of glucose. Here, we synthesized AuPt bimetallic nanoparticles with unique surface electron structure due to the coupling effect of the two metal components, achieving improved glucose catalytic oxidase. Because of the effective work function difference between the two metals in AuPt, the electrons will transfer from Au to accumulate on Pt, simultaneously contributing to the substantial enhancement of Au-induced glucose oxidase and Pt-induced catalase performance. We systematically studied the enzyme-catalytic efficiency of AuPt with varied two metal proportions, in which Au:Pt at 3:1 showed the highest catalytic efficiency of glucose oxidase in solution. The AuPt nanoparticles were further co-cultured with cells and also showed excellent biological activity for glucose oxidase. This work demonstrates that the physicochemical properties between different metals can be exploited for engineering high-performance metal nanoparticle-based nanozymes, which opens up a new way to rationally design and optimize artificial nanozymes to mimic natural enzymes.

Keywords: AuPt alloys, bimetallic nanozymes, electron transfer, catalytic activity, rational design

\section{INTRODUCTION}

Natural enzymes as biocatalysts, mediate almost every biological process in living, but their inherent disadvantages such as high cost, easy inactivation, and difficult to recover, largely limit application in biomedical engineering (Wolfenden and Snider, 2001; Pietrzak and Ivanova, 2021). This inspires scientists to explore artificial substitutes for enzymes (Wang et al., 2020). Many nanomaterials so far have been found with significant enzyme-like catalytic activities, such as $\mathrm{Fe}_{3} \mathrm{O}_{4}$ nanoparticles (NPs) and carbon nanotubes with peroxidase (POD-like) properties, and Pt NPs and $\mathrm{CeO}_{2} \mathrm{NPs}$ with catalase (CAT-like) activities, which are commonly referred to as nanozymes (Gao et al., 2007; Huang et al., 2019). As an alternative to natural enzymes, nanozymes possess characteristics such as low cost, high stability, easy mass production, and adjustable activity, and show enormous potentials in a wide range of applications, including biosensing, imaging, food manufacturing, and pollution prevention (Dong et al., 2012; Fan et al., 2012; Tian et al., 2020). However, low catalytic efficiency is still one key issue facing in practical applications (Chen et al., 2021).

Gold nanoparticles (Au NPs) with glucose oxidase (GOx-like) features have yielded promising responses in biomedical application (Lin et al., 2014; Wu et al., 2018; Zhang et al., 2019a). In the 
relevant studies of structure-performance of Au nanozymes, a variety of parameters including size and shape, surface structure, local composition and chemical bonding have been proved to play important impacts on their catalytic performances (Zhou et al., 2010; Hakkinen, 2012; Shen et al., 2015; Li et al., 2019; LouFranco et al., 2020). In fact, during catalytic process, the oxidation-redox reactions take place just on the surface of $\mathrm{Au}$ $\mathrm{NPs}$, realized by the electron transfer between $\mathrm{Au}$ and the surrounding reactants (Della Pina et al., 2008). Therefore, regulating the physicochemical properties of Au NPs is vitally required for effective modulation of electron transfer on surface, so as to boost their intrinsic biomimetic catalytic ability (Jiao et al., 2019). On account of the effective work function difference between different composites, it offers the possibility to modulate the electron density of $\mathrm{Au}$ at the atomic scale, which may favor the fast mass transport and electron transfer during the catalytic process (Yamauchi et al., 2012). Therefore, the design of Au with other composites is greatly expected to be an effective way to develop Au nanozymes with excellent performance, and even may bring unprecedented insights into the relationship between electron density of Au and enzyme-like activities.

Herein, AuPt bimetallic alloys were synthesized as effective nanozymes for the catalytic oxidation of glucose. Since the work function of Au was less than that of Pt in AuPt alloys, the electrons on $\mathrm{Au}$ will flow to accumulate on $\mathrm{Pt}$, making $\mathrm{Pt}$ electron-rich. This rearrangement of electrons in $\mathrm{AuPt}$ contributes to enhanced catalytic oxidation performance of $\mathrm{Au}$ for $\mathrm{GOx}$, as well as catalytic reduction of $\mathrm{H}_{2} \mathrm{O}_{2}$ by $\mathrm{Pt}$, thus giving 1.4-fold GOx-like activity improvement over Au NPs alone. AuPt alloys showed negligible cytotoxicity at a certain concentration, but significantly affected the energy metabolism process of cells due to the consumption of glucose. It was expected to use AuPt bimetallic nanozymes for subsequent biological researches, such as treatment of cancer or diabetes (Nosrati et al., 2021).

\section{MATERIALS AND METHODS}

\section{Materials}

$\mathrm{HAuCl}_{4} \cdot 3 \mathrm{H}_{2} \mathrm{O}$ ( $\geq 49.0 \%$ Au basis), $\mathrm{H}_{2} \mathrm{PtCl}_{6} \cdot 6 \mathrm{H}_{2} \mathrm{O}(\geq 37.50 \% \mathrm{Pt}$ basis), hydrogen peroxide $\left(\mathrm{H}_{2} \mathrm{O}_{2}, 30 \%\right)$ and Methyl thiazolyl tetrazolium (MTT, 98\%) were purchased from Sigma-Aldrich (Louis, MO, United States). Glucose was purchased from Macklin. Trifluoroacetic acid $\left(\mathrm{CF}_{3} \mathrm{COOH}, 99 \%\right)$ was purchased from Alfa Aesar. The ATP assay kit, Enhanced BCA protein assay kit and Calcein/PI cell viability assay kit were from Beyotime Institute of Biotechnology, China. All chemical agents in this work were utilized without further purification. The Milli-Q water was obtained from the Milli-Q System.

\section{Characterization of AuPt Alloys}

The transmission electron microscope (TEM) images of the nanoparticles were obtained on an FEI Tecnai G2 F30 microscope. The crystalline phases of the materials were collected with X-ray powder diffraction (XRD; Rigaku Ultima IV), using copper Ka radiation $(\lambda=0.154056 \mathrm{~nm})$ in the $2 \theta$ range of $20^{\circ}-90^{\circ}$ at a scan rate of $20^{\circ} / \mathrm{min}$. Fourier transform infrared spectroscopy (FT-IR) spectra was measured by a BRUKER TENSOR II in the range of $4,000-400 \mathrm{~cm}^{-1}$. Dynamic light scattering (DLS) particle size analyzer (Malvern, United States) was used to measure the hydrophilic diameters of the particles. The concentration of $\mathrm{Au}$ and $\mathrm{Pt}$ was measured by Agilent Technologies 5100 inductively coupled plasma optical emission Spectrometry (ICP-OES). Shimadzu, AXIS SUPRA was used to detect X-ray photoelectron spectroscopy (XPS). The position of the $\mathrm{C} 1 \mathrm{~s}$ peak at $284.8 \mathrm{eV}$ was used as a calibration reference to determine the accurate binding energies. UV-vis absorption spectra was recorded on Shimadzu UV 3600 plus. The oxygen content in water was measured by dissolved oxygen tester JPSJ-605F, Leici China. The confocal laser scanning microscopy (CLSM) images were obtained using NIKON A1 R.

\section{Synthesis of AuPt Alloys}

We added $5 \mathrm{ml}$ of $\mathrm{HAuCl}_{4} \cdot 3 \mathrm{H}_{2} \mathrm{O}(20 \mathrm{mmol})$ and $\mathrm{H}_{2} \mathrm{PtCl}_{6} \cdot 6 \mathrm{H}_{2} \mathrm{O}$ $(20 \mathrm{mmol})$ in different volume ratios to $95 \mathrm{ml}$ water, stirred it for 5 min until it was evenly mixed, and then heated the mixture to boiling. Then, $10 \mathrm{ml}$ sodium citrate aqueous solution with a concentration of $20 \mathrm{mg} / \mathrm{ml}$ was added to the boiling liquid and stirred for $10 \mathrm{~min}$. Next, turned off the heat and continued to stir vigorously to room temperature. AuPt alloys with different proportions were obtained by centrifugal washing. Au NPs and Pt NPs were synthesized by similar methods. The composition of AuPt alloys was determined by ICP-OES.

\section{Glucose Oxidation Reaction}

The catalytic performance of the alloys was characterized by the formation of gluconic acid. $50 \mu \mathrm{L}$ AuPt alloys $(2 \mathrm{mg} / \mathrm{ml})$ and $950 \mu \mathrm{L}$ glucose $(1 \mathrm{M})$ were mixed evenly for $4 \mathrm{~h}$ to verify the effect of AuPt alloys on glucose catalytic oxidation. After the reaction, AuPt alloys were removed by centrifugation to obtain supernatant containing gluconic acid.

Solution A (5 mM EDTA and $0.15 \mathrm{mM}$ triethylamine aqueous solution), solution $\mathrm{B}$ ( $3 \mathrm{M}$ hydroxylamine, $\mathrm{NH}_{2} \mathrm{OH}$ ), and solution $\mathrm{C}\left(1 \mathrm{M} \mathrm{HCl}, 0.1 \mathrm{M} \mathrm{FeCl}\right.$ and $0.25 \mathrm{M} \mathrm{CCl}_{3} \mathrm{COOH}$ aqueous solution) were respectively prepared. Then, $250 \mu \mathrm{L}$ solution $\mathrm{A}$ and $25 \mu \mathrm{L}$ solution $\mathrm{B}$ were successively added to the clear liquid. After $25 \mathrm{~min}$ of incubation, $125 \mu \mathrm{L}$ solution $\mathrm{C}$ was added, and the mixture was evenly mixed for $5 \mathrm{~min}$ before spectral test (Huang et al., 2017).

\section{Catalyze Decomposition of $\mathrm{H}_{2} \mathrm{O}_{2}$}

$10 \mu \mathrm{L}$ AuPt alloys $(2 \mathrm{mg} / \mathrm{ml})$ were mixed with an aqueous solution of $\mathrm{H}_{2} \mathrm{O}_{2}(10 \mathrm{mM})$, with a total volume of $5 \mathrm{ml}$, and the amount of oxygen produced by the decomposition of $\mathrm{H}_{2} \mathrm{O}_{2}$ was recorded by a dissolved oxygen recorder (Luo et al., 2021). All the reactions were carried out in deoxidized water at $37{ }^{\circ} \mathrm{C}$.

\section{Cell Viability Assessment for AuPt Alloys}

In order to evaluate the cell compatibility of AuPt alloys, PC12 cells were seeded into 96-well cell culture plates at $1 \times 10^{4} /$ well for $24 \mathrm{~h}$. Then $\mathrm{Au}_{0.75} \mathrm{Pt}_{0.25}$ alloys with different concentrations were added into 96-well plates and cultured for $24 \mathrm{~h}$. After the culture 

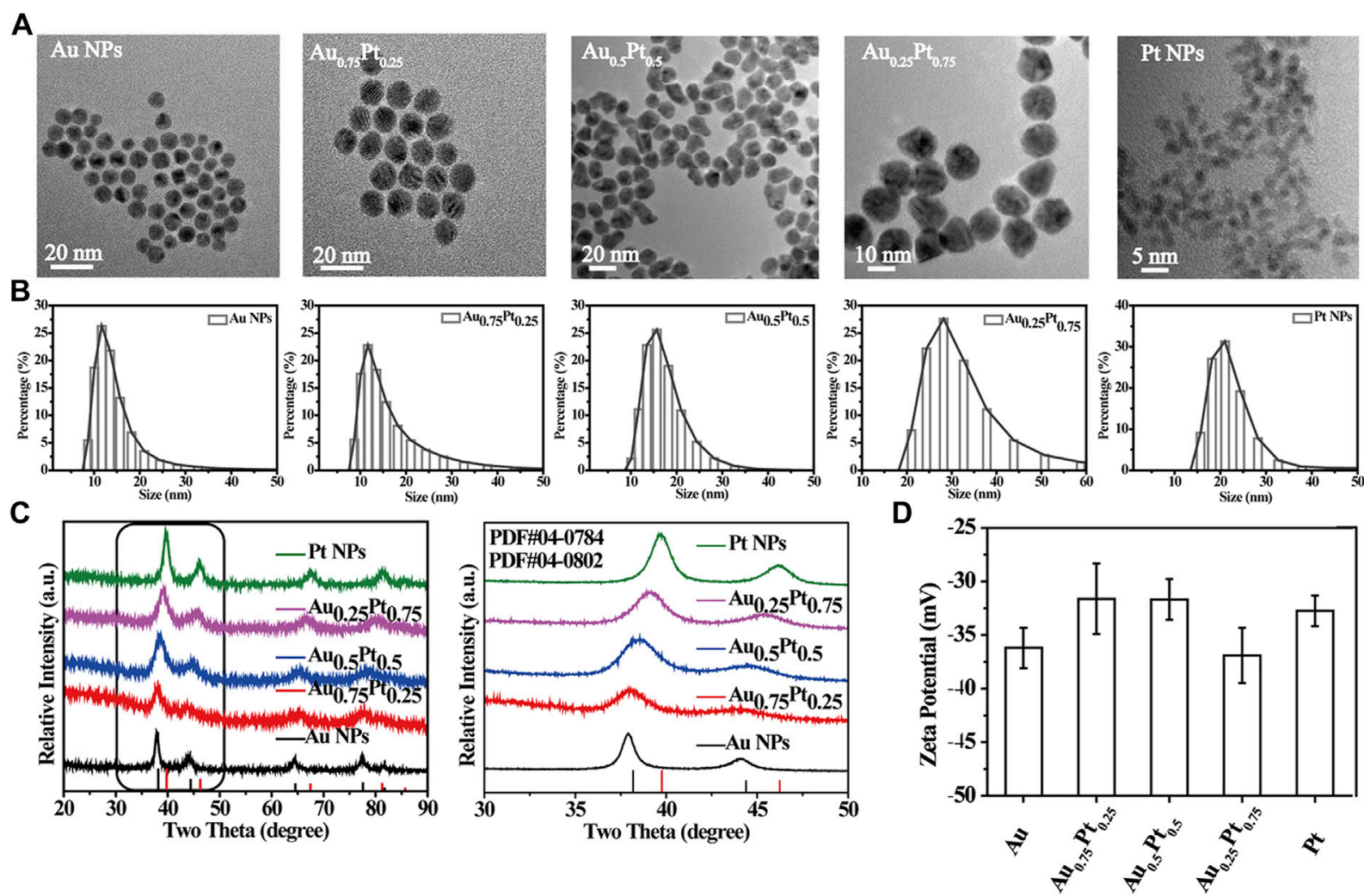

FIGURE 1 | Characterization of the prepared AuPt alloys. (A) TEM images of AuPt alloys with different proportions. (B) Hydrodynamic diameter distribution of AuPt alloys with different proportions. (C) XRD patterns of AuPt alloys with different proportions, partial enlarged XRD patterns. (D) Zeta potential of AuPt alloys with different proportions

medium was sucked out, $100 \mu \mathrm{L}$ MTT (phosphate buffered saline (PBS) buffer solution $0.6 \mathrm{mg} / \mathrm{ml}$ ) was added to each well and cultured for $4 \mathrm{~h}$. Finally, removed the MTT solution, added $100 \mu \mathrm{L}$ dimethyl sulfoxide (DMSO), and incubated for $10 \mathrm{~min}$. The absorbance of the solution at $490 \mathrm{~nm}$ was measured with a microplate reader.

\section{Confocal Laser Scanning Microscopy Imaging}

PC12 cells were inoculated in $1 \mathrm{ml} \mathrm{CLSM} \mathrm{dish} \mathrm{at} 1 \times 10^{5} / \mathrm{ml}$ and incubated overnight to ensure firm cell adhesion. The cells were incubated with AuPt alloys of different concentrations for $24 \mathrm{~h}$, and then the cells in the corresponding groups were dyed alive and dead with Calcein (green) and PI (red).

\section{Intracellular ATP Detection}

PC12 cells were inoculated at a density of $5 \times 10^{5}$ cells/well and incubated in 6-well plates for $12 \mathrm{~h}$. Then AuPt alloys with different proportions were added and incubated for $24 \mathrm{~h}$. After that, the cells were lysed with ATP lysis buffer, and the supernatant was collected by centrifugation. The protein concentration of each sample was determined by BCA protein concentration kit, and ATP concentration was detected by ATP detection kit (Liu et al., 2021).

\section{RESULTS AND DISCUSSIONS}

\section{Characterization of Material Properties}

AuPt alloys with different proportions were prepared by one-step reduction of $\mathrm{AuCl}_{4}{ }^{-}$and $\mathrm{PtCl}_{6}{ }^{2-}$ with sodium citrate in water. As shown in Figure 1A, TEM images showed that these AuPt alloys with different proportions were uniformly spherical. High resolution TEM (HRTEM) images and the corresponding FAST Fourier transform (FFT) further revealed the lattice plane of the $\mathrm{Au}_{0.75} \mathrm{Pt}_{0.25}$ alloys (Supplementary Figure S1). The calculated lattice spacing of the (111) plane of the alloys was $0.230 \mathrm{~nm}$, which was between the lattice spacing of the pure $\mathrm{Au}(111)$ plane $(0.235 \mathrm{~nm})$ and that of the pure Pt (111) plane $(0.228 \mathrm{~nm})$. The lattice spacing in the middle proved the formation of AuPt alloys (He et al., 2017).

In addition, the average hydrodynamic diameters of AuPt alloys synthesized with different AuPt ratios by DLS test were approximately 10-20 $\mathrm{nm}$ and had good dispersibility (Figure 1B) (Zhang et al., 2019b). The stability of the $\mathrm{Au}_{0.75} \mathrm{Pt}_{0.25}$ was further evaluated. TEM images showed that the morphological characteristics of $\mathrm{Au}_{0.75} \mathrm{Pt}_{0.25}$ did not change significantly after 3 days of soaking in PBS (Supplementary Figure S2), indicating that $\mathrm{Au}_{0.75} \mathrm{Pt}_{0.25}$ had excellent stability. The Zeta potential was about $-30 \mathrm{mV}$ due to the $-\mathrm{COOH}$ in sodium citrate on the surface of the AuPt alloys (Figure 1D). Supplementary Figure S3 


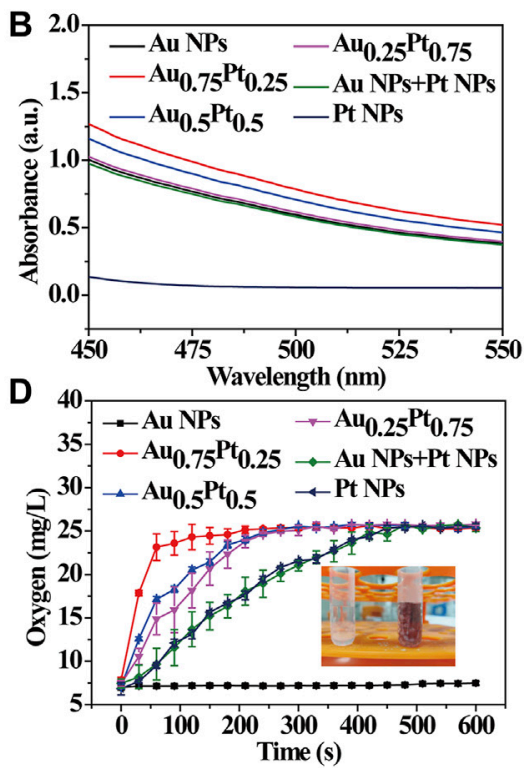

FIGURE 2 | Catalytic activity test of AuPt alloys. (A) Schematic illustration of catalytic oxidation of glucose in AuPt Alloys. (B) UV-vis absorption spectra of AuPt alloys with different proportions incubation with glucose for $4 \mathrm{~h}$. AuPt alloys catalyzed the oxidation of glucose to gluconic acid, which is mixed with hydroxylamine and $\mathrm{Fe}^{3+}$ to produce a red complex. (C) Absorbance value at $505 \mathrm{~nm}$ determined by a colorimetric assay. (D) Dissolved oxygen level of AuPt alloys with different proportions solution after incubation with $\mathrm{H}_{2} \mathrm{O}_{2}$.

showed the characteristic peaks of sodium citrate, such as $-\mathrm{C}=\mathrm{O}$ characteristic absorption peak at $1,600 \mathrm{~cm}^{-1}$ and $-\mathrm{CH}_{2}$ shear vibration peak at $1,400 \mathrm{~cm}^{-1}$, indicating the successful preparation of sodium citrate stabilized AuPt alloys (Sanches et al., 2011; Zhang et al., 2018).

It was well known that the lattice of two metals would change after they formed alloys, and their diffraction patterns would change correspondingly according to the basic principle of crystal diffraction. Therefore, XRD pattern was used to further confirm the formation of AuPt alloys. Figure 1C showed XRD patterns of alloys with different proportions. Diffraction peaks of $\mathrm{Au}, \mathrm{Pt}$ and AuPt alloys with different AuPt ratios indicated that the prepared samples all had face-centered cubic (fcc) phases. According to Figure 1C, the $2 \theta$ peaks (111) and (200) of AuPt NPs fell between the corresponding $2 \theta$ peaks of $\mathrm{Au}$ and Pt NPs (Liu et al., 2011). With the change of AuPt ratios, the positions of these peaks changed gradually, indicating the change of alloying degree. UV-vis absorption spectrum (Supplementary Figure S4) also confirmed the formation of AuPt alloys. With the gradual addition of $\mathrm{Pt}$ component, the typical absorption of Au NPs at $520 \mathrm{~nm}$ due to surface plasmon resonance would gradually disappear. The vanishing peak also represented the gradual formation of $\mathrm{AuPt}$ alloys (Zhao et al., 2014).

The relative abundance of $\mathrm{Au}$ and $\mathrm{Pt}$ in AuPt alloys detected by ICP and XPS was shown in Supplementary Table S1. Because the standard redox potential $\mathrm{E}^{0}$ for $\mathrm{AuCl}_{4}{ }^{-} / \mathrm{Au}^{0}$ couple $(+0.99 \mathrm{~V})$ was higher than that of $\mathrm{PtCl}_{6}{ }^{2-} / \mathrm{Pt}^{0}$ couple $(+0.74 \mathrm{~V})$, the Au was reduced first (De and Rao, 2005). The percentage of Au element in the alloys obtained from ICP was slightly higher than that in the raw solution, and the results of XPS measurement further confirmed that Pt element was enriched on the surface of the alloys.

\section{AuPt Alloys for Glucose Catalytic Oxidation and Decomposition of $\mathrm{H}_{2} \mathrm{O}_{2}$}

Since the prepared AuPt alloys included different catalytic properties, in which $\mathrm{Au}$ components possessed the intrinsic GOx-like activity (Luo et al., 2010), while Pt components owned the intrinsic CAT-like activity [Figure 2A(1)] (Fan et al., 2011), it was expected that AuPt alloys could catalyze the cascade reaction of glucose oxidation [Figure $2 \mathbf{A}(2)$ ]. To prove it, the GOx-like activity of AuPt alloys was first investigated. AuPt alloys in different proportions reacted with $\mathrm{O}_{2}$ in the reaction solution to catalyze the oxidation of glucose, resulting in $\mathrm{H}_{2} \mathrm{O}_{2}$ and gluconic acid. The reaction solution was centrifuged to remove AuPt alloys, and the supernatant obtained contained $\mathrm{H}_{2} \mathrm{O}_{2}$ and gluconic acid. In a typical experiment, with gluconic acid as substrate, $\mathrm{NH}_{2} \mathrm{OH}$ and $\mathrm{Fe}^{3+}$ were successively added to the solution, and the reaction of glucose to gluconic acid catalyzed by AuPt alloys were detected by a colorimetric assay. As shown in Figure 2B, the apparent absorbance band at 450-550 nm confirmed that gluconic acid was the product of glucose oxidation catalyzed by AuPt alloys. Next, we made statistics on the absorbance value at $505 \mathrm{~nm}$ (Figure 2C), and it could be seen that $\mathrm{Au}_{0.75} \mathrm{Pt}_{0.25}$ had the best catalytic effect compared with other alloys and physical mixing group. The above experimental results confirmed the GOx-like activity of the AuPt alloys. 

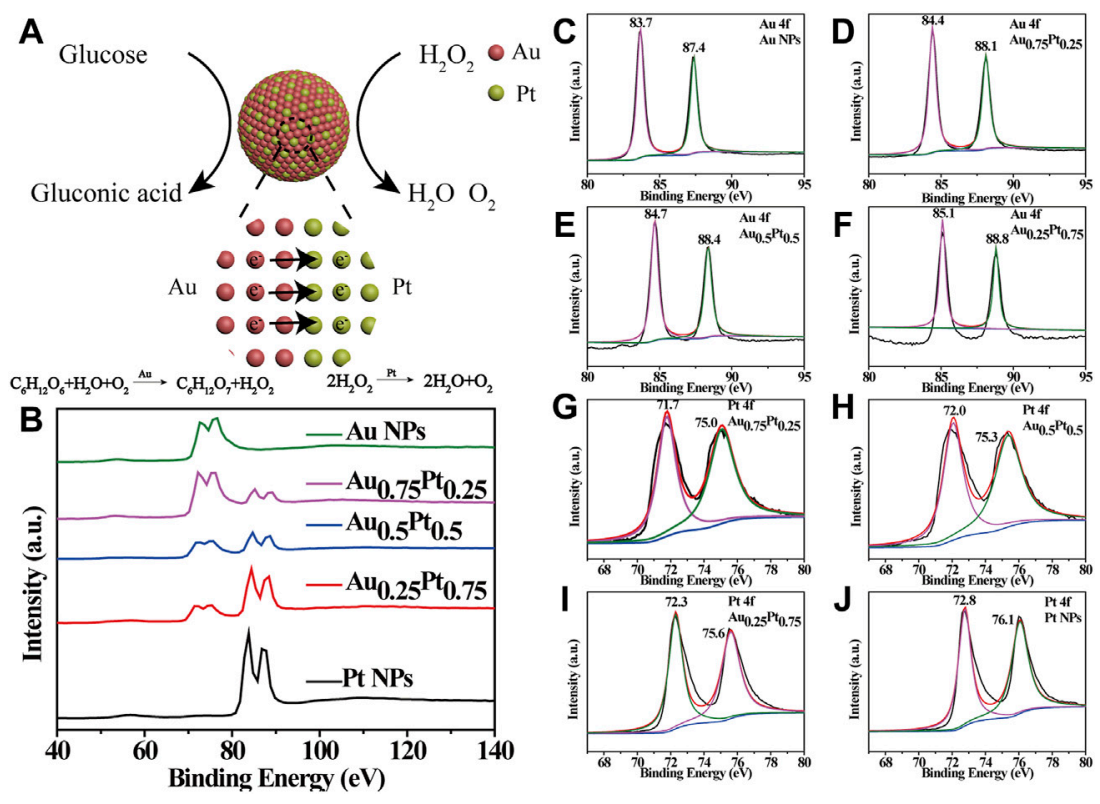

FIGURE 3 | Electron distribution states in AuPt alloys. (A) Schematic illustration of electron transfer between Au and Pt. (B) Partial XPS spectrum of AuPt alloys with different proportions. Show the representative $\mathrm{Au} 4 \mathrm{f}$ of the (C) Au NPs, (D) $\mathrm{Au}_{0.75} \mathrm{Pt}_{0.25}$, (E) $\mathrm{Au}_{0.5} \mathrm{Pt}_{0.5}$, and (F) $\mathrm{Au}_{0.25} \mathrm{Pt}_{0.75}$. Show the representative Pt $4 f$ of the (G) $\mathrm{Au}_{0.75} \mathrm{Pt}_{0.25}$, (H) $\mathrm{Au}_{0.5} \mathrm{Pt}_{0.5}$, (I) $\mathrm{Au}_{0.25} \mathrm{Pt}_{0.75}$ and (J) Pt NPs.

Then, the CAT-like activity of AuPt alloys was studied. Since it could catalyze $\mathrm{H}_{2} \mathrm{O}_{2}$ to produce $\mathrm{H}_{2} \mathrm{O}$ and $\mathrm{O}_{2}$, we explored the catalytic process of AuPt alloys for $\mathrm{H}_{2} \mathrm{O}_{2}$ by testing the content of dissolved oxygen. A large number of bubbles were observed in the tubes containing $\mathrm{H}_{2} \mathrm{O}_{2}$ after the addition of AuPt alloys in different proportions (except pure Au NRs), demonstrating that $\mathrm{Pt}$ in AuPt alloys could play a CAT-like effect (Figure 2D). Next, we used a solution-oxygen meter to further monitor the oxygen generation process and found that $\mathrm{Au}_{0.75} \mathrm{Pt}_{0.25}$ catalyzed the decomposition of $\mathrm{H}_{2} \mathrm{O}_{2}$ to generate oxygen at the fastest rate.

As mentioned above, AuPt alloys had GOx-like and CAT-like activities, and different AuPt ratios exhibited different nanozymes catalytic activities, among which $\mathrm{Au}_{0.75} \mathrm{Pt}_{0.25}$ had the best catalytic effect.

\section{Electron States in AuPt Alloys}

To further explore the reasons for the enhancement of the catalytic activity of AuPt alloys as nanozymes, we conducted the following analysis. We realized that it has been reported that the electronic structure of metal nanoparticles played a critical role in their catalytic activity (Duchesne et al., 2018). Therefore, we investigated whether the alloying of $\mathrm{Au}$ and $\mathrm{Pt}$ would change the electronic structures of $\mathrm{Au}$ and $\mathrm{Pt}$ components, thus affecting the catalytic performance (Figure 3A). The electron binding energies of XPS for $\mathrm{Au}$ and Pt in AuPt alloys with different proportions were used to reflect the charge distribution of AuPt in alloys (Supplementary Figure S5). Among them, in AuPt alloys system, the peak between 60 and $90 \mathrm{eV}$ corresponded to Pt $4 \mathrm{f}_{7 / 2}$, Pt $4 \mathrm{f}_{5 / 2}$ and $\mathrm{Au} 4 \mathrm{f}_{7 / 2}, \mathrm{Au} 4 \mathrm{f}_{5 / 2}$, respectively (Figure 3B) (Yamauchi et al., 2012). As shown in Figure 3C, the binding energy of $\mathrm{Au} 4 \mathrm{f}$ in $\mathrm{Au}$ NPs was 83.7 and $87.4 \mathrm{eV}$, respectively. With the addition of $\mathrm{Pt}$ component, the $4 \mathrm{f}$ binding energy of $\mathrm{Au}$ component increased in AuPt alloys (Figures 3D-F). However, the $4 \mathrm{f}$ binding energy of Pt component in Pt NPs was 72.8 and $76.1 \mathrm{eV}$ (Figure 3J), and the binding energy shifted to lower with the decrease of Pt component content (Figures 3G-I). The above analysis results proved that with the formation of AuPt alloys, the electron on $\mathrm{Au}$ component in $\mathrm{AuPt}$ alloys transfered to $\mathrm{Pt}$ component, resulting in lower electron density of $\mathrm{Au}$ component in AuPt alloys than Au NPs, and higher electron density of Pt component than Pt NPs.

Based on the above interesting results, we provided a theoretical basis for the change of electronic structure of AuPt alloys with different components. For bimetallic alloys, the work function of different metals was the decisive factor affecting the regulation of electronic structure. Then, for AuPt alloys, the work functions of $\mathrm{Au}$ and $\mathrm{Pt}$ were 5.54 and $6.13 \mathrm{eV}$, respectively. The difference in the work function between $\mathrm{Au}$ and Pt components caused the electrons on Au surface to be transfer towards Pt. Coincidentally, in the current study on the mechanism of $\mathrm{Au}$ nanozymes catalyzing glucose oxidation, Au components needed to maintain positive valence, and Pt surface was rich in electrons, which was conducive to its catalase properties. Therefore, electron transfer due to work function would synergistically enhance the effect of the two components of AuPt as nanozymes.

\section{In vitro Performance of AuPt Alloys}

In order to be applied in subsequent biological studies, we evaluated the biological activity of AuPt alloys. MTT assay (Figure 4B) was used to verify the toxicity of PC12 cells cocultured with AuPt alloys, and it was found that AuPt alloys had 


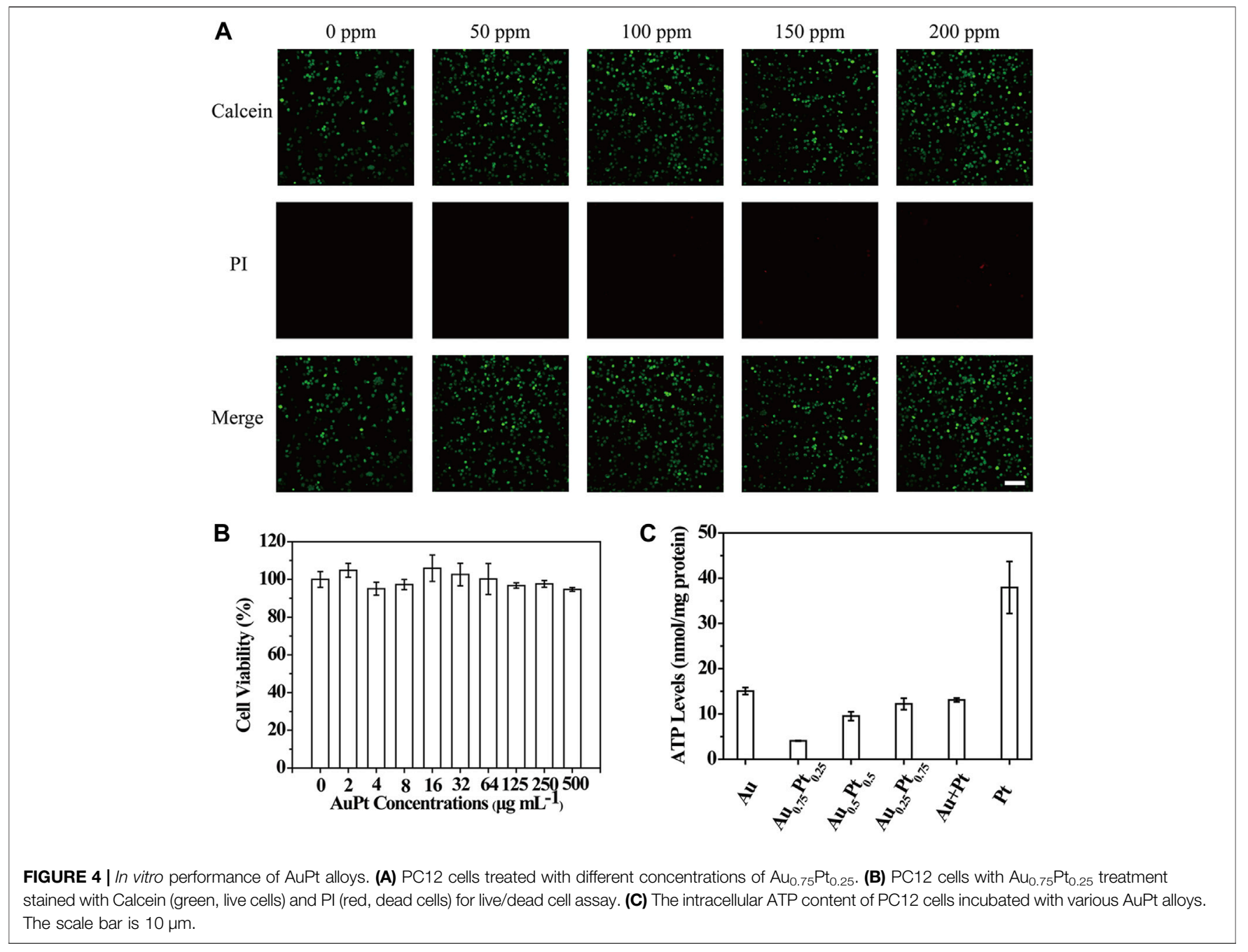

no obvious toxicity for cells. Subsequently, the survival of cells cocultured with AuPt alloys for $24 \mathrm{~h}$ was observed in confocal images by live/dead staining experiment. Cells were stained with calcein/PI, in which living cells were stained green with calcein and dead cells were stained red with PI. Confocal images (Figure 4A) could be observed that AuPt alloys materials with different concentrations had no obvious killing effect on cells, which also facilitated the application of AuPt alloys for further research.

Encouraged by the high catalytic performance of AuPt alloys in aqueous solution for glucose and $\mathrm{H}_{2} \mathrm{O}_{2}$, we carried out cell level experiments to observe the catalytic effect of AuPt alloys. We verified whether AuPt alloys could affect the cellular metabolism process because glucose was the main energy source for cell activity. Intracellular ATP levels were measured using ATP detection kits (Supplementary Figures S6, S7). As shown in Figure 4C, by catalyzing glucose oxidation, AuPt alloys could effectively reduce intracellular ATP levels by affecting energy supply. And it was worth noting that $\mathrm{Au}_{0.75} \mathrm{Pt}_{0.25}$ also had the best catalytic effect at the cellular level. Therefore, AuPt alloys, which affected cell energy metabolism, were considered as a treatment for glucose-related diseases.

\section{CONCLUSION}

In summary, our work reported the synthesis of AuPt alloys with varied molar ratios of AuPt via one-step reduction method in aqueous solution. This method provides a useful reference for the preparation of other binary or multicomponent metal nanoparticles. Since the large difference of the work function between $\mathrm{Au}$ and $\mathrm{Pt}$, the electron transfer effect would occur in AuPt alloys, promoting the electron flow from the lower work function of $\mathrm{Au}$ to the higher work function side of Pt. Compared with pure $\mathrm{Au}$ and $\mathrm{Pt}$, the alloying nanozymes showed higher activities of GOx-like and CAT-like. In addition, AuPt alloys showed low cytotoxicity in vitro but could affect the glucose metabolism due to its catalytic oxidation of glucose, holding great promise in the further development for clinic disease diagnosis and treatment. 


\section{DATA AVAILABILITY STATEMENT}

The original contributions presented in the study are included in the article/Supplementary Material, further inquiries can be directed to the corresponding author.

\section{AUTHOR CONTRIBUTIONS}

FC and TS designed the project and performed the experiments. FC prepared the figures and drafted the manuscript. All authors discussed the paper and the submitted version.

\section{REFERENCES}

Chen, Y., Wang, P., Hao, H., Hong, J., Li, H., Ji, S., et al. (2021). Thermal Atomization of Platinum Nanoparticles into Single Atoms: An Effective Strategy for Engineering High-Performance Nanozymes. J. Am. Chem. Soc. 143, 18643-18651. doi:10.1021/jacs.1c08581

De, G., and Rao, C. N. R. (2005). Au-Pt alloy Nanocrystals Incorporated in Silica Films. J. Mater. Chem. 15, 891-894. doi:10.1039/b412429d

Della Pina, C., Falletta, E., Prati, L., and Rossi, M. (2008). Selective Oxidation Using Gold. Chem. Soc. Rev. 37, 2077-2095. doi:10.1039/b707319b

Dong, Z., Luo, Q., and Liu, J. (2012). Artificial Enzymes Based on Supramolecular Scaffolds. Chem. Soc. Rev. 41, 7890-7908. doi:10.1039/c2cs35207a

Duchesne, P. N., Li, Z. Y., Deming, C. P., Fung, V., Zhao, X., Yuan, J., et al. (2018). Golden Single-Atomic-Site Platinum Electrocatalysts. Nat. Mater 17, 1033-1039. doi:10.1038/s41563-018-0167-5

Fan, J., Yin, J.-J., Ning, B., Wu, X., Hu, Y., Ferrari, M., et al. (2011). Direct Evidence for Catalase and Peroxidase Activities of Ferritin-Platinum Nanoparticles. Biomaterials 32, 1611-1618. doi:10.1016/j.biomaterials.2010.11.004

Fan, K., Cao, C., Pan, Y., Lu, D., Yang, D., Feng, J., et al. (2012). Magnetoferritin Nanoparticles for Targeting and Visualizing Tumour Tissues. Nat. Nanotech. 7, 459-464. doi:10.1038/nnano.2012.90

Gao, L., Zhuang, J., Nie, L., Zhang, J., Zhang, Y., Gu, N., et al. (2007). Intrinsic Peroxidase-like Activity of Ferromagnetic Nanoparticles. Nat. Nanotech. 2, 577-583. doi:10.1038/nnano.2007.260

Häkkinen, H. (2012). The Gold-Sulfur Interface at the Nanoscale. Nat. Chem 4, 443-455. doi:10.1038/nchem.1352

He, W., Han, X., Jia, H., Cai, J., Zhou, Y., and Zheng, Z. (2017). AuPt Alloy Nanostructures with Tunable Composition and Enzyme-like Activities for Colorimetric Detection of Bisulfide. Sci. Rep. 7, 40103. doi:10.1038/srep40103

Huang, Y., Zhao, M., Han, S., Lai, Z., Yang, J., Tan, C., et al. (2017). Growth of Au Nanoparticles on 2D Metalloporphyrinic Metal-Organic Framework Nanosheets Used as Biomimetic Catalysts for Cascade Reactions. Adv. Mater. 29, 1700102. doi:10.1002/adma.201700102

Huang, Y., Ren, J., and Qu, X. (2019). Nanozymes: Classification, Catalytic Mechanisms, Activity Regulation, and Applications. Chem. Rev. 119, 4357-4412. doi:10.1021/acs.chemrev.8b00672

Jiao, L., Xu, W., Yan, H., Wu, Y., Gu, W., Li, H., et al. (2019). A Dopamine-Induced $\mathrm{Au}$ Hydrogel Nanozyme for Enhanced Biomimetic Catalysis. Chem. Commun. 55, 9865-9868. doi:10.1039/c9cc04436a

Li, M., Lao, Y.-H., Mintz, R. L., Chen, Z., Shao, D., Hu, H., et al. (2019). A Multifunctional Mesoporous Silica-Gold Nanocluster Hybrid Platform for Selective Breast Cancer Cell Detection Using a Catalytic AmplificationBased Colorimetric Assay. Nanoscale 11, 2631-2636. doi:10.1039/c8nr08337a

Lin, Y., Ren, J., and Qu, X. (2014). Nano-gold as Artificial Enzymes: Hidden Talents. Adv. Mater. 26, 4200-4217. doi:10.1002/adma.201400238

Liu, J., Cao, L., Huang, W., and Li, Z. (2011). Preparation of AuPt alloy Foam Films and Their superior Electrocatalytic Activity for the Oxidation of Formic Acid. ACS Appl. Mater. Inter. 3, 3552-3558. doi:10.1021/am200782x

Liu, P., Zhou, Y., Shi, X., Yuan, Y., Peng, Y., Hua, S., et al. (2021). A Cyclic NanoReactor Achieving Enhanced Photodynamic Tumor Therapy by Reversing Multiple Resistances. J. Nanobiotechnol. 19, 149. doi:10.1186/s12951-021-00893-6

\section{FUNDING}

This research was funded by the China Postdoctoral Science Foundation (Grant No. BX20200245, 2020M681397).

\section{SUPPLEMENTARY MATERIAL}

The Supplementary Material for this article can be found online at: https://www.frontiersin.org/articles/10.3389/fchem.2022.854516/ full\#supplementary-material

Lou-Franco, J., Das, B., Elliott, C., and Cao, C. (2020). Gold Nanozymes: From Concept to Biomedical Applications. Nano-Micro. Lett. 13, 10. doi:10.1007/ s40820-020-00532-Z

Luo, W., Zhu, C., Su, S., Li, D., He, Y., Huang, Q., et al. (2010). Self-Catalyzed, SelfLimiting Growth of Glucose Oxidase-Mimicking Gold Nanoparticles. ACS. Nano. 4, 7451-7458. doi:10.1021/nn102592h

Luo, T., Wang, D., Liu, L., Zhang, Y., Han, C., Xie, Y., et al. (2021). Switching Reactive Oxygen Species into Reactive Nitrogen Species by Photocleaved O 2 Released Nanoplatforms Favors Hypoxic Tumor Repression. Adv. Sci. 8, 2101065. doi:10.1002/advs.202101065

Nosrati, H., Aramideh Khouy, R., Nosrati, A., Khodaei, M., Banitalebi-Dehkordi, M., Ashrafi-Dehkordi, K., et al. (2021). Nanocomposite Scaffolds for Accelerating Chronic Wound Healing by Enhancing Angiogenesis. J. Nanobiotechnol. 19, 1. doi:10.1186/s12951-020-00755-7

Pietrzak, M., and Ivanova, P. (2021). Bimetallic and Multimetallic Nanoparticles as Nanozymes. Sensors Actuators B: Chem. 336, 129736. doi:10.1016/j.snb.2021. 129736

Sanches, E. A., Soares, J. C., Iost, R. M., Marangoni, V. S., Trovati, G., Batista, T., et al. (2011). Structural Characterization of Emeraldine-Salt Polyaniline/ Gold Nanoparticles Complexes. J. Nanomater. 2011, 1-7. doi:10.1155/2011/ 697071

Shen, X., Liu, W., Gao, X., Lu, Z., Wu, X., and Gao, X. (2015). Mechanisms of Oxidase and Superoxide Dismutation-like Activities of Gold, Silver, Platinum, and Palladium, and Their Alloys: A General Way to the Activation of Molecular Oxygen. J. Am. Chem. Soc. 137, 15882-15891. doi: $10.1021 /$ jacs.5b10346

Tian, R., Xu, J., Luo, Q., Hou, C., and Liu, J. (2020). Rational Design and Biological Application of Antioxidant Nanozymes. Front. Chem. 8, 831. doi:10.3389/ fchem.2020.00831

Wang, P., Wang, T., Hong, J., Yan, X., and Liang, M. (2020). Nanozymes: A New Disease Imaging Strategy. Front. Bioeng. Biotechnol. 8, 15. doi:10.3389/fbioe 2020.00015

Wolfenden, R., and Snider, M. J. (2001). The Depth of Chemical Time and the Power of Enzymes as Catalysts. Acc. Chem. Res. 34, 938-945. doi:10.1021/ $\operatorname{ar} 000058 \mathrm{i}$

Wu, J., Qin, K., Yuan, D., Tan, J., Qin, L., Zhang, X., et al. (2018). Rational Design of Au@Pt Multibranched Nanostructures as Bifunctional Nanozymes. ACS Appl. Mater. Inter. 10, 12954-12959. doi:10.1021/acsami.7b17945

Yamauchi, Y., Tonegawa, A., Komatsu, M., Wang, H., Wang, L., Nemoto, Y., et al. (2012). Electrochemical Synthesis of Mesoporous Pt-Au Binary Alloys with Tunable Compositions for Enhancement of Electrochemical Performance. J. Am. Chem. Soc. 134, 5100-5109. doi:10.1021/ja209044g

Zhang, K., Cheng, Y., Ren, W., Sun, L., Liu, C., Wang, D., et al. (2018). Coordination-Responsive Longitudinal Relaxation Tuning as a Versatile MRI Sensing Protocol for Malignancy Targets. Adv. Sci. 5, 1800021. doi:10. 1002/advs.201800021

Zhang, K., Fang, Y., He, Y., Yin, H., Guan, X., Pu, Y., et al. (2019a). Extravascular Gelation Shrinkage-Derived Internal Stress Enables Tumor Starvation Therapy with Suppressed Metastasis and Recurrence. Nat. Commun. 10, 5380. doi:10. 1038/s41467-019-13115-3

Zhang, K., Li, H. Y., Lang, J. Y., Li, X. T., Yue, W. W., Yin, Y. F., et al. (2019b). Quantum Yield-Engineered Biocompatible Probes Illuminate Lung Tumor 
Based on Viscosity Confinement-Mediated Antiaggregation. Adv. Funct. Mater. 29, 1905124. doi:10.1002/adfm.201905124

Zhao, Y., Ye, C., Liu, W., Chen, R., and Jiang, X. (2014). Tuning the Composition of AuPt Bimetallic Nanoparticles for Antibacterial Application. Angew. Chem. Int. Ed. 53, 8127-8131. doi:10.1002/ange.20140103510.1002/anie.201401035

Zhou, X., Xu, W., Liu, G., Panda, D., and Chen, P. (2010). Size-Dependent Catalytic Activity and Dynamics of Gold Nanoparticles at the Single-Molecule Level. J. Am. Chem. Soc. 132, 138-146. doi:10.1021/ja904307n

Conflict of Interest: The authors declare that the research was conducted in the absence of any commercial or financial relationships that could be construed as a potential conflict of interest.
Publisher's Note: All claims expressed in this article are solely those of the authors and do not necessarily represent those of their affiliated organizations, or those of the publisher, the editors, and the reviewers. Any product that may be evaluated in this article, or claim that may be made by its manufacturer, is not guaranteed or endorsed by the publisher.

Copyright $(2022$ Chen and Song. This is an open-access article distributed under the terms of the Creative Commons Attribution License (CC BY). The use, distribution or reproduction in other forums is permitted, provided the original author $(s)$ and the copyright owner(s) are credited and that the original publication in this journal is cited, in accordance with accepted academic practice. No use, distribution or reproduction is permitted which does not comply with these terms. 